\title{
Comparison of Differential Evolution and Particle Swarm Optimization for Optimizing the Power Generation Potential in Hydroelectric Power Plants using Dot Net
}

\author{
Dipti Shiphrah Navaraj ${ }^{1}$, B. Priya Esther ${ }^{2}$, Nishant David Solomon ${ }^{3}$ \\ ${ }^{I}$ PG Scholar Department of Electrical and Electronics Engineering AMC Engineering College Bangalore- \\ 560083 \\ ${ }^{2}$ Associate Professor Department of Electrical and Electronics Engineering AMC Engineering College \\ Bangalore-560083 \\ ${ }^{3}$ Senior Software Engineer
}

\begin{abstract}
This paper proposes an optimization model for the selection of turbines in order to improve the power generation potential in a Hydro Power Plant. The thermal, electrical and mechanical stresses that occur over time on the turbine affect the efficiency of the generating unit. The mathematical formulation for this problem emanates a non-linear programming problem which is therefore solved using Particle Swarm Optimization and Differential Evolution optimizing techniques. These two algorithms are then compared and the results are validated in Dot Net. The Dot Net framework is used due to its extensive security, enhanced usability and flexibility. These optimization techniques are implemented in a case study which is carried out in Murudeshwar Small Hydro Power Plant located in Narayanpur, Bijapur District, India.
\end{abstract}

Keywords: Hydroelectric Power Plant (HPP), Particle Swarm Optimization (PSO), Differential Evolution $(D E)$, Generating Unit (GU), Dot Net, Hours of Permanence.

\section{Introduction}

Hydro is the largest renewable energy source utilized for Power Generation. India is immensely blessed with abundant hydroelectric potential and ranks $5^{\text {th }}$ in terms of exploitable potential on a global scenario. The energy policy of India is largely defined by the country's expanding energy deficit and increased focus on developing alternative energy sources. India also ranks fourth in terms of energy consumption after China, USA, and Russia. Due to this increase in energy consumption, there is a copious requirement of power which can be brought about by installing new hydroelectric power plants or the existing power plants can be repowered.

The efficiency of a Generating Unit depends on performance of the turbine. The natural process of aging of a GU in a Hydroelectric Power Plant is foreseeable. The GU's performance will decrease over time due to the wear and tear of the turbine, which may include pitting due to cavitations, cracking and abrasion of the turbine due to suspended particles in the water [1]. Thus, the selection of turbine plays a vital role in the efficiency of a Generating Unit which in turn affects the power generation potential in a Hydroelectric Power Plant [2]. The selection of the turbine efficiency curves that would be ideal for each operation system leads to a mixed, non-linear programming problem. Modern heuristic optimization techniques based on operational research and artificial intelligence concepts, such as Genetic Algorithm[3],[4], Particle Swarm Optimization[5], Artificial Bee Colony[6], Bacterial Foraging Algorithm[7][8], Ant Lion[9],[10], NSGA-II [11],[12], Big BangBig Crunch[13],[14], Grey Wolf Optimization [15],[16]and Differential Evolution [17] provide outstanding solutions for the non-linear nature of the real world problems. Each method has its own merits and de-merits. However, Differential Evolution (DE) gained popularity as the best suitable solution algorithm for such problems due to its rate of convergence, robustness and a very minute or negligible percentage error. The results are analyzed and verified in Dot Net and the best turbine is displayed.

\section{Turbine Characteristics in brief}

In order to select the best turbine for a particular hydro site, a few factors need to be taken into consideration. They are, site characteristics, the available water head, turbine rotational speed and flow discharge. There are mainly two types of turbines, which are: Impulse turbine and Reaction turbine.

The impulse turbine generally makes use of the velocity of the water to move the runner and discharges to atmospheric pressure. The stream of water hits each bucket on the runner. There is no suction below the turbine, and the water flows out the bottom of the turbine housing after hitting the runner. An impulse turbine is generally suitable for high head and low flow applications. The types of Impulse turbines are Pelton and Crossflow. A reaction turbine develops power from the combined action of pressure and moving water. The runner is placed directly in the water stream flowing over the blades rather than striking each individually. Reaction 
turbines are generally used for sites having low heads and large amount of water flow. The types of Reaction Turbines are Francis, Kaplan and Propeller.

The Francis turbine is the oldest turbine, which has a rotational speed between 50 and 500rpm and can be mainly installed for power plants having an available head of $30 \mathrm{~m}$ to $600 \mathrm{~m}$. The Propeller turbine which is also known as Semi-Kaplan Turbine has a rotational speed of 200-1000 rpm. This kind of turbine is usually installed in HPP's having an available head of 3m-80m. The Full Kaplan has a turbine rotational speed of 500 and $1000 \mathrm{rpm}$ and is installed in plants having heads between $8 \mathrm{~m}$ and $60 \mathrm{~m}$. Pelton has a turbine rotational speed of 4 and $70 \mathrm{rpm}$ and usually installed in high head power plants having heads above $300 \mathrm{~m}$.

\begin{tabular}{|c|l|c|c|}
\hline Type of Turbine & Class & Head (m) & $\begin{array}{c}\text { Rotational Speed } \\
(\mathbf{r p m})\end{array}$ \\
\hline Propeller & Reaction & $3-80$ & $200-1000$ \\
\hline Kaplan & Reaction & $8-60$ & $500-1000$ \\
\hline Francis & Reaction & $30-600$ & $50-500$ \\
\hline Pelton & Impulse & Above 300 & $4-70$ \\
\hline
\end{tabular}

Table I: Characteristics of the turbines

The selection of the type of turbine used for a particular power plant is mainly based on the available head and turbine rotational speed. Every turbine has a different efficiency curve type. This is shown in Fig 1 [19]

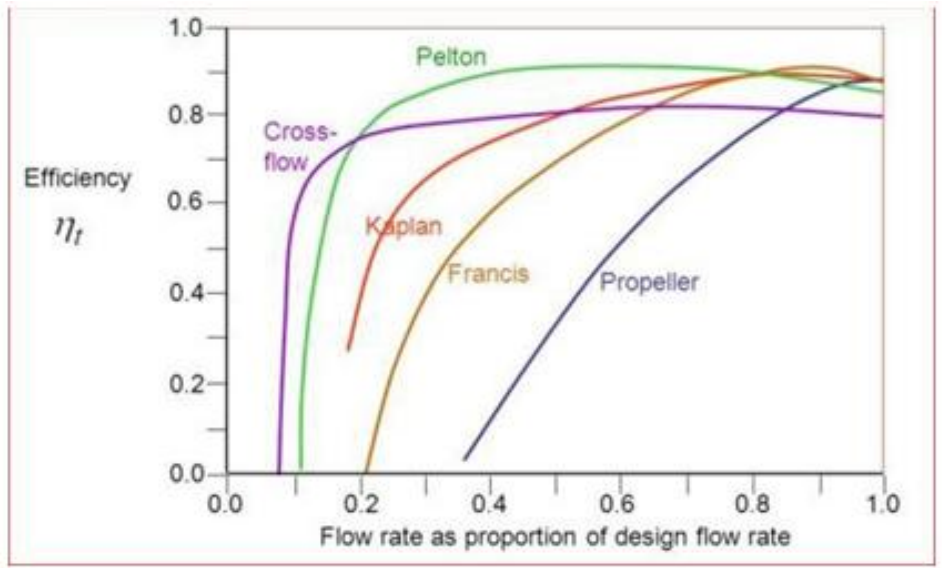

Fig.1: Efficiency curves of the different types of turbines [19]

In this paper the load levels are split into three categories. They are considered as: light, intermediate and heavy loads. The load levels aim to represent the demand in each load level, the hours of permanence of each load and the efficiency of each turbine at that particular load. It is important to choose efficiency curve types that consider an adequate balance between load and permanence time.

\section{Problem Formulation}

The problem is to optimize the potential of generation of power by the generating unit in a new or repowered hydro electric power plant. The mathematical formulation of this problem is as shown below.

$$
\operatorname{Max} \frac{\sum_{l} C^{l} H^{l} D^{l}}{\sum_{l} C^{l} H^{l} \sum_{j} \sum_{k} \frac{y_{j}^{l, k} g_{j}^{l}}{\eta\left(g_{j}^{l}\right)}}
$$

Subject to $\quad \sum_{j} \sum_{k} y_{j}^{l, k} g_{j}^{l}=D^{l}$

$\sum_{k} y_{j}^{l, k} \underline{G}^{k} \leq g_{j}^{l} \leq \sum_{k} y_{j}^{l, k} \bar{G}^{k}$ 


$$
\begin{aligned}
& y_{j}^{l, k} \leq z_{j}^{k} \\
& \sum_{k} z_{j}^{k}=1 \\
& y_{j}^{l, k}, z_{j}^{k} \in\{0,1\}
\end{aligned}
$$

Equation (1) is a Maximization Function which represents the total generation efficiency of the Hydro Plant. The function takes into account $l$ levels of load (i.e., heavy, intermediate and light load levels), subjective to the number of hours of permanence $H^{l}$ and energy $\operatorname{cost} C^{l}$ for each load level.

$H^{l} D^{l}$ in equation (1) represents the total energy generated in the HPP at load level $l \cdot y_{j}^{l, k} g_{j}^{l} / \eta^{k}\left(g_{j}^{l}\right)$ is the total output used by the $j^{\text {th }}$ generating unit to generate $g_{j}^{l}$. $D^{t}$ represents the total demand of the HPP which is calculated by the sum of all the productivities of the GU.

In the denominator of the objective function, we see that the total output is multiplied by $H^{l}$ which has the sum in energy. Together, the equation is therefore multiplied by the energy $\operatorname{cost} C^{l}$.

The objective function is subjected to a certain constraint which is given by equation (2). Here, the total demand of the HPP at load level $l$ is given by the total generation of each unit at load level $l$ represented by $g_{j}^{l}$ which is multiplied by the dispatch variable $y_{j}^{l, k}$.

The upper and lower generating limits of each GU is represented by equation (3). If a GU assumes a curve type to be $k$, the dispatch variable $y_{j}^{l, k}$ can have a value of 1 or 0 . This is represented by equation (4). Finally equation (5) represents the GU's selected efficiency curve type [20].

The mathematical formulation results in a non-linear programming problem. This is due to the non-linearity occurring between the available head and the flow discharge in a Hydroelectric Power Plant. This problem is solved using PSO and DE Algorithms [21].

\subsection{Particle Swarm Optimization (PSO)}

\section{Optimization Techniques}

Particle Swarm Optimization was developed in the year 1995 by James Kennedy and Russell Eberhart. It is a population based metaheuristic algorithm based on the analogy with swarms of birds. In PSO, every particle or bird (or agent) is considered as a solution which is characterized by its position and velocity [22]. The flowchart of a general PSO algorithm is as shown in Fig 2. 


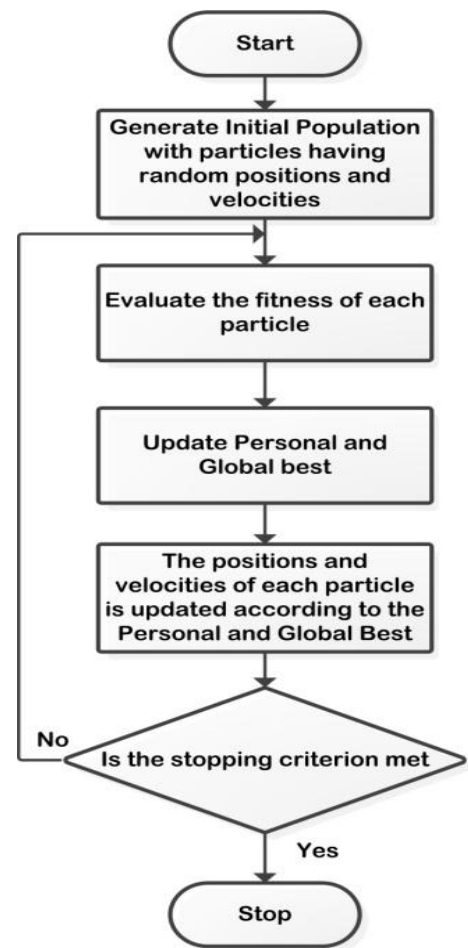

Fig 2: A Flowchart of Particle Swarm Optimization Algorithm

In PSO, an ancestor particle generates a new particle, according to "movement rule" seen in equations (7) and (8)

$$
\begin{aligned}
& X_{p}^{t+1}=X_{p}^{t}+V_{p}^{t+1} \\
& V_{p}^{t+1}=V_{p}^{t}+\operatorname{Rnd}_{1} \cdot\left(b_{p}-X_{p}^{t}\right)+R n d_{2} \cdot\left(b_{G}-X_{p}^{t}\right) \cdot c f
\end{aligned}
$$

the expressions $X_{p}^{t}, X_{p}^{t+1}$ are the positions of the particles $p$ in iterations $t$ and $t+1, V_{p}^{t}, V_{p}^{t+1}$ are the velocity vectors of the particle $p$ from iteration $t-1$ to it and from $t$ to $t+1, R n d_{1}$ and $R n d_{2}$ are random numbers ranging between 0 and 1 , and $b_{G}$ is the global best particle [19]. A few advantages of using PSO are that it is simple to understand, easy to implement and has less computation time.

\subsection{Differential Evolution}

Differential evolution was developed in the year 1996 by Raine Storn and Kenneth Price, a year after particle swarm optimization was introduced. As PSO showed powerful outcomes and the various advantages it had over the existing algorithms, DE was left unexplored. DE has gained popularity in the power system field only in the recent years and is now being considered over PSO as it has many advantages over PSO hence making it a remarkable evolutionary algorithm. Differential evolution is a metaheuristic search algorithm that maximizes/ minimizes a given objective function $f(\operatorname{var} 1, \operatorname{var} 2, \ldots, \operatorname{var} N)$. For $N$ variable function, the possible solutions are represented by an $N$ dimensional vector $x$, where each parameter of $x$ represents one of the function variables shown in equation (9)

$f(\operatorname{var} 1, \operatorname{var} 2, \ldots \operatorname{var} N)=f\left(x_{1}, x_{2}, \ldots, x_{N}\right)$

A population refers to a set of $N P$ vectors. Every valid vector $x$ in population $P$ is referred to as a member of $P$ [23].

$P=\{x(1), x(2), \ldots, x(N P)\}$

A generation is a new population that is developed from a parent population which is acquired through recombination and selection. Recombination creates an offspring population from the parent population. Selection chooses which parents and offspring move on to a new population that will become the parent population for the next generation [24]. A flowchart explaining the basics of Differential Evolution Algorithm is provided in Fig. 3. 


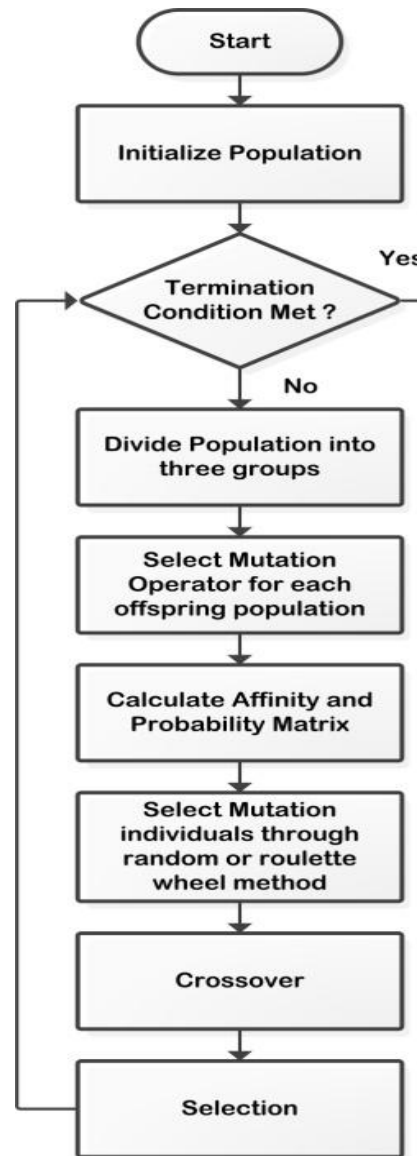

Fig 3: Flowchart of Differential Evolution

\section{Case Study}

A case study is carried out at Murudeshwar Power Corporation which is a Small Hydroelectric Power Plant located in Bijapur District, India to verify the performance of the proposed optimal methods. The HPP consists of two generating units of 5.8 MW rating each. The turbine that is currently being used for both the generating units is a horizontal Full Kaplan turbine. The data used for this case study is from July 2013February 2014, a year which experienced a particularly active rainy season.

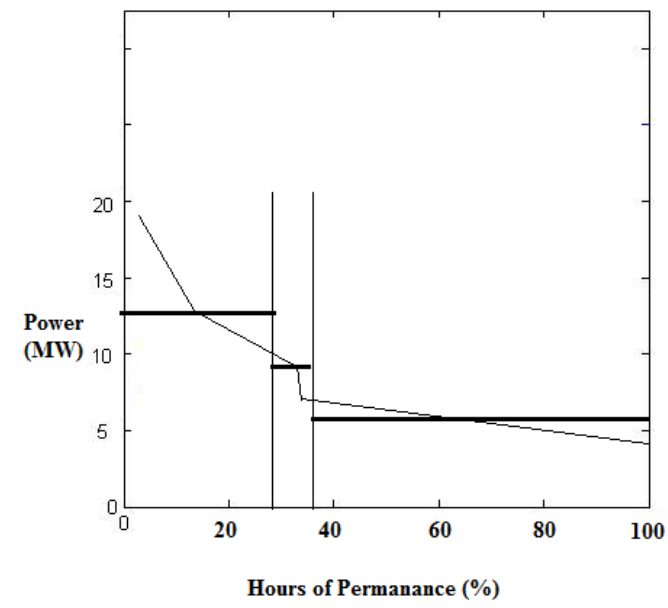

Fig 4: Duration of Load for the year 2013-2014

The data for the Load Duration Curve and the Energy Price was provided by the HPP as shown in Fig.4. Table II and Table III consists of the Kaplan and Propeller turbine data. 
Table II: Murudeshwar HPP (Kaplan)

\begin{tabular}{|l|l|l|l|}
\hline Year 2013 & & Murudeshwar HPP & \\
\hline & Heavy & Intermediate & Light \\
\hline Load (MW) & 13.3227 & 6.0736 & 3.8725 \\
\hline Hours of Permanence (\%) & 28.70 & 48.87 & 22.42 \\
\hline Price (Rs./MWh) & 33.77 & 74.09 & 116.204 \\
\hline
\end{tabular}

Table III: Murudeshwar HPP (Propeller)

\begin{tabular}{|l|l|l|l|}
\hline Year 2013 & & Murudeshwar HPP & \\
\hline & Heavy & Intermediate & Light \\
\hline Load (MW) & 14.1727 & 9.8895 & 5.9945 \\
\hline Hours of Permanence (\%) & 29.032 & 6.45 & 64.51 \\
\hline Price (Rs./MWh) & 42.33 & 60.67 & 100.09 \\
\hline
\end{tabular}

\section{Results}

The implementation of the algorithms is carried out in Dot Net. Security, flexibility and great user experience -put together, define a Dot Net Framework.

The most valuable advantage of using Dot Net is its simplicity and compatibility with different programming languages. For example, Visual Basic and $\mathrm{C}++$ are complex languages, which can be carried out in Dot Net. Another advantage of the Microsoft Dot Net Framework is that is can be easily integrated into multiple formats. In this paper, the language used for coding is $\mathrm{C}++$. The implementation using $\mathrm{C}++$ coding was simple and effective in particular Framework.

\subsection{Particle Swarm Optimization}

When PSO algorithm is developed for the non-linear mathematical problem mentioned, it shows remarkable results. The accuracy level is up to 10 decimal points and the value converges within a few iterations. For simulation, the parameters taken into consideration were:

- Iterations: 1000

- Dimension:2

- Swarm Length:5

The results displayed in Fig 5 are the best evaluation found for both Kaplan and Propeller turbine. Comparing the two values, it is evident that a Propeller turbine is more suitable for this particular case study.
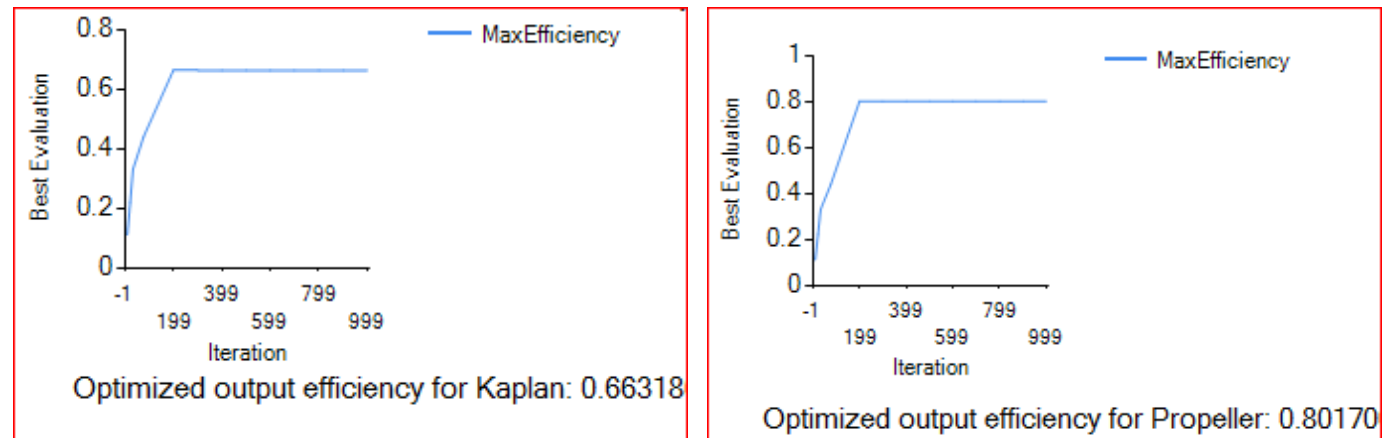

Fig 5: The best evaluation found for Kaplan and Propeller turbine using PSO

\subsection{Differential Evolution}

On the implementation of Differential Evolution algorithm, the results obtained validate the predictions of the algorithm. The most important observation is that the percentage error calculated for DE is very less or negligible. The parameters considered are as follows:

- Iterations :5000

- Population length: 50

- Mutation rate: 20

From the results shown in Fig6, it is evident that a Propeller turbine would be more suitable. A Propeller turbine has higher efficiency than a Kaplan turbine and also is cost effective. 


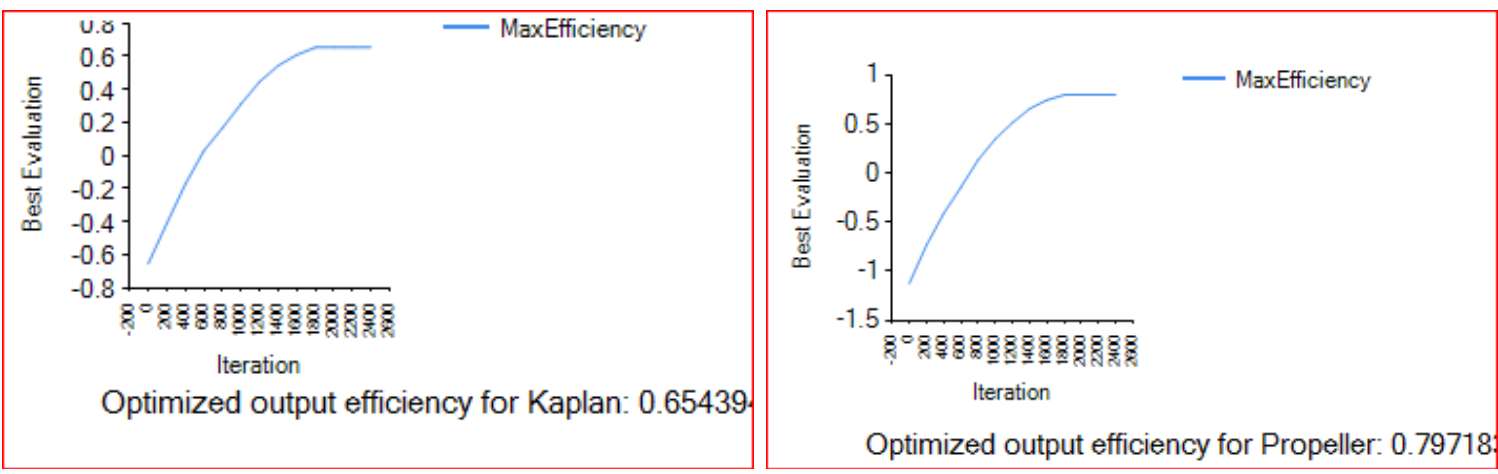

Fig 6: The best evaluation found for Kaplan and Propeller turbine using DE

\section{Conclusion}

The optimization of power generation potential was carried out using Particle Swarm Optimization and Differential Evolution algorithms. The results were compared and validated in Dot Net. The experimental results prove that DE outperforms PSO. DE is robust, highly accurate, has a high convergence rate and negligible percentage error. The simulation results show that a Propeller turbine can be used to repower the Murudeshwar Small HPP in order to increase the power generation potential. A Propeller turbine is very efficient and cost effective. Further research in this field can be done with a more efficient algorithm like Grey Wolf Optimization Algorithm which has a higher accuracy level.

\section{References}

[1]. Padhy, M.K., and Sainia, R.P. (2008). "A review on silt erosion in hydroturbines". Renewable and Sustainable Energy Reviews, 12(7), 1974-1987.

[2]. Soares, S.; Salmazo, C. T. (1997). "Minimum loss predispatch model for hydroelectric power plant systems." IEEE Transactions on PowerSystems, v. 12, n. 3, p. $1220-1228$

[3]. Dorothea Heiss-Czedik.(1997). “An Introduction to Genetic Algorithms.” Artificial Life, v 3, Issue 1, p 63-65.

[4]. G.S Sampaio, J.T. Saraiva, J.C Sousa, V.T Mendes.(2013). "Optimization of the Operation of Hydro Stations in Market Environment Using Genetic Algorithms." 10 ${ }^{\text {th }}$ International Conference on the European Energy Market (EEM), p 1-8.

[5]. Said. M. Mikki, Ahmed. A. Kishk.(2008). "A PSO: A Physics-Based Approach." Synthesis Lectures on Computational Electromagnetics, p 103

[6]. D. Karaboga, B. Basturk. (2008). " On the performance of Artificial Bee Colony (ABC) Algorithm.” Elsevier, Applied Soft Computing, v 8, Issue 1, p 687-697.

[7]. B.Priya Esther; KS Krishna; KS Kumar; K Ravi (2016). “ Demand Side Management using Baterial Foraging Optimization Algorithm." Information Systems Design and Intelligent Applications v. 433 of the series Advances in Intelligent Systems and Computing p. 657-666.

[8]. B. Priya Esther; K. Satish Kumar (2016). "A survey on Residential Demand Side Management architecture, approaches, optimization models and methods." Renewable and Sustainable Energy Reviews, v.59, p.342-351.

[9]. Seyedali Mirjalili.(2015). "The Ant Lion Optimizer.” Elsevier, Advance in Engineering Software, v 83, p 80-98.

[10]. Hari Mohan Dubey, Manjaree Pandit, B.K Panigrahi.(2016). "Ant Lion Optimization for short-term wind integrated hydrothermal power generation scheduling.” Elsevier, International Journal of Electrical Power and Energy System, v 83, p 158-174.

[11]. Kalyanmoy Deb, Amrit Pratap, Sameer Agarwal, T. Meyarivan.(2002). "A Fast and Elitist Multiobjective Genetic Algorithm NSGA-II.” IEEE Transaction on Evolutionary Computation, v 6, p 182-197.

[12]. Azadeh Kamjoo, Alireza Maheri, Arash M. Dizqah, Ghanim A. Putrus.(2016). "Multi-objective design under uncertainties of hybrid Renewable Energy Sources using NSGA-II and chance constrained programming." Elsevier, International Journal of Electrical Power and Energy System, v 74, p 187-194.

[13]. Osman K. Erol, Ibrahim Eksin.(2006). “A New Optimization method: Big Bang- Big Crunch.” Elsevier, Advances in Engineering Software, v 37, p 106-111.

[14]. C Fatih Kucuktezcan, V.M Istemihan Genc.(2015). "Preventive and Corrective Control applications in power systems via Big Bang- Big Crunch optimization.” Elsevier, International Journal of Electrical Power and Energy System, v 67, p 114-124.

[15]. Seyedali Mirjalili, Seyed Mohammad Mirijalili, Andrew Lewis.(2014). " Grey Wolf Optimizer.” Elsevier, Advances in Engineering Software, v 69, p 46-61.

[16]. N. Jayakumar, S. Subramanian, S. Ganesan, E. B. Elanchezhian.(2016). "Grey Wolf Optimization for combined heat and power dispatch with cogeneration systems.” Elsevier, International Journal of Electrical Power and Energy System, v 74, p 252-264.

[17]. Chengfo Sun, Haiyan Zhou, Liqing Chen.(2012). "Improved Differential Evolution Algorithms." IEEE International Computer Science and Automation Engineering (CSAE), v 3, p 142-145.

[18]. C. Jalivu, I. Visa, D. Diaconescu, R. Saulescu, M. Neagoe, O. Climescu. (2010). “ Dynamic Model of a Small Hydropower Plant.” $12^{\text {th }}$ International Conference on Optimization of Electrical and Electronic Equipment (OPTIM), p 1216-1223.

[19]. http://images.slideplayer.com/1/330622/slides/slide_40.jpg

[20]. Jéssica Pillon Torralba Fernandes, Paulo de Barros Correia, Ieda Geriberto Hidalgo, Glauber Renato Colnago. (2013). “ A Genetic Algorithm Solution for Optimization of the Power Generation Potential in Hydroelectric Plants.'IEEE Conference on Evolutionary Computation, p 2504-2511.

[21]. Amita Mahor, Vishnu Prasad, Saroj Rangnekar.(2009). "Scheduling of Cascaded Hydro Power System: A New Self Adaptive Inertia Weight Particle Swarm Optimization Approach." International Conference on Advances in Recent Technologies in Communication and Computing, p 565-570. 
[22]. André S Pacheco, João Tomé Saraiva (2014). “An Evolutionary Particle Swarm Optimization, EPSO, Approach to Optimize the Operation of Hydro Stations in Market Environment." $11^{\text {th }}$ International Conference on the European Energy Market (EEM14), p16.

[23]. Joel H. Van Sickel, Kwang Y.Lee, Jin S.Heo.(2007). "Differential Evolution and its Applications to Power Plant Control." International Conference on Intelligent Systems Application to Power Systems(ISAP), p 1-6.

[24]. Gao-yang Li, Ming-guang Lin.(2010). "The summary of Differential Evolution Algorithm and its Improvement." $3^{\text {rd }}$ International Conference on Advanced Computer Theory and Engineering (ICACTE), v3, p153-156. 